\title{
Fear Deficits in Hypomyelinated Tppp Knock-Out Mice
}

\author{
Huy Nguyen, ${ }^{1}$ Lindsey M. Meservey, ${ }^{2}$ Nao Ishiko-Silveria, ${ }^{2}$ Mu Zhou, ${ }^{3}$ Ting-Ting Huang, ${ }^{1,4}$ and \\ Meng-meng $\mathrm{Fu}^{2}$
}

https://doi.org/10.1523/ENEURO.0170-20.2020

${ }^{1}$ Department of Neurology, Stanford University School of Medicine, Stanford, CA 94305, ${ }^{2}$ Department of Neurobiology, Stanford University School of Medicine, Stanford, CA 94305, ${ }^{3}$ Department of Molecular and Cellular Physiology, Stanford University School of Medicine, Stanford, CA 94305, and ${ }^{4}$ Geriatric Research, Education, and Care Center, VA Palo Alto Health Care System, Palo Alto, CA 94304

\begin{abstract}
Oligodendrocytes in the central nervous system (CNS) produce myelin sheaths that insulate axons to facilitate efficient electrical conduction. These myelin sheaths contain lamellar microtubules that enable vesicular transport into the inner sheath. Mechanistically, oligodendrocytes rely on Golgi outpost organelles and the associated protein tubulin polymerization promoting protein (TPPP) to nucleate or form new microtubules outside of the cell body. Consequently, elongation of lamellar microtubules is defective in Tppp knock-out (KO) mice, which have thinner and shorter myelin sheaths. We now explore the behavioral phenotypes of Tppp KO mice using a number of different assays. In open-field assays, Tppp KO mice display similar activity levels and movement patterns as wild-type mice, indicating that they do not display anxiety behavior. However, Tppp KO mice lack fear responses by two types of assays, traditional fear-conditioning assays and looming fear assays, which test for innate fear responses. Deficits in fear conditioning, which is a memory-dependent task, as well as in spatial memory tests, support possible short-term memory defects in Tppp KO mice. Together, our experiments indicate a connection between CNS myelination and behavioral deficits.
\end{abstract}

Key words: fear; microtubule; myelin; oligodendrocyte

\section{Significance Statement}

Oligodendrocytes are cells in the brain that make myelin sheaths, which wrap concentrically around axons to provide insulation that facilitates electrical conduction. Fear responses have historically been attributed to neuronal activity. However, emerging literature indicates that mouse models with defective myelination display long-term fear deficits. Here, we look at a specific mouse model that lacks an oligodendrocyte-specific protein that is important for building the cellular structure of microtubules, which allow for transport to and along the myelin sheath. These mice display deficits in both memory-dependent fear as well as innate fear responses. Thus, our work indicates that myelin structure is important for fear response.

\section{Introduction}

Oligodendrocytes insulate neuronal axons in a lipid-rich covering called myelin that facilitates fast axonal conduction velocities. Defects in myelination contribute to diseases, including multiple sclerosis and leukodystrophies. In addition, faulty myelination has been previously im-

Received April 29, 2020; accepted July 9, 2020; First published September 2, 2020.

The authors declare no competing financial interests. plicated in psychiatric diseases, including schizophrenia, depression, bipolar disorder, and obsessive-compulsive disorder (Fields, 2008).

Both neuroimaging and genetics data connect white matter changes with schizophrenia, a mental disorder that can manifest in hallucinations and delusions. Magnetic resonance

Author contributions: H.N., T.-T.H., and M.-m.F. designed research; H.N. and N.I.-S. performed research; H.N., L.M.M., M.Z., and M.-m.F. analyzed data; H.N., L.M.M., T.-T.H., and M.-m.F. wrote the paper. 
imaging (MRI) studies have indicated a disruption in white matter structure in schizophrenic patients (Foong et al., 2000; Kubicki et al., 2005; Fields, 2008). In addition, polymorphisms associated with schizophrenia are found in genes encoding oligodendrocyte transcription factor 2 (OLIG2; Georgieva et al., 2006), which is crucial for oligodendrocyte development, and 2',3'-cyclic nucleotide 3'-phosphodiesterase (CNP), a structural myelin protein (Hagemeyer et al., 2012).

Many studies of human schizophrenic brains have observed changes to myelin or oligodendrocytes. Electron microscopy (EM) of prefrontal cortex (PFC) of schizophrenic brains indicates damage to myelin sheath lamellae and changes in oligodendrocyte organelle content (Uranova et al., 2001). The frontal gyrus of schizophrenic patients contains $\sim 20 \%$ fewer oligodendrocytes than those of control patients (Hof et al., 2002). Multiple microarray studies of both prefrontal and temporal cortex of schizophrenic brains found significant downregulation of genes involved in myelination and oligodendrocyte development (Hakak et al., 2001; Tkachev et al., 2003; Aston et al., 2004). These studies raise the intriguing hypothesis that defects in oligodendrocyte development and myelination may underlie symptoms in schizophrenia.

In mice, several recent studies suggest a link between myelination defects and behavioral phenotypes. Mice exposed to cuprizone, a demyelinating agent, exhibit deterioration of oligodendrocytes in the PFC and schizophrenialike symptoms (Gregg et al., 2009). Mass spectrometry experiments elucidated transient changes in myelin proteins $\sim 24 \mathrm{~h}$ following contextual fear conditioning (Houyoux et al., 2017), indicating that myelin remodeling may be involved in short-term fear response.

Evidence from genetic mouse models suggest that enhanced myelination heightens fear-conditioned learning while impaired myelination impedes fear-conditioned learning. For example, activation of ERK1/2 (extracellular signal-regulated kinase) in oligodendrocytes increased myelination throughout the CNS and increased contextual response after fear conditioning (Jeffries et al., 2016). On the other hand, mice in which the transcription factor

This work was supported by the Dr. Miriam and Sheldon G. Adelson Medical Research Foundation (AMRF) Grant 100005984 (to Ben A. Barres, M.-m.F. and L.M.), the Vincent J. Coates Research Foundation (to Ben A. Barres, M.-m.F. and L.M.), the Myra Reinhard Foundation (to Ben A. Barres, M.-m.F. and L.M.), the Department of Defense CDMRP Grant GW160094 (to H.N. and T.-T.H.), and the Department of Veterans Affairs Merit Review Grant BX-0024-71 (to H.N. and T.-T.H.).

Acknowledgements: We thank the Andrew Huberman Lab for advice and use of their looming fear assay apparatus (Heekyung Jung, Lindsay Salay, Nao Ishiko-Silveria); the Thomas Sudhof Lab (Mu Zhou, Karthik Raju) for advice and use of their fear conditioning assay apparatus; Alex Valenzuela for technical assistance with animal husbandry; and Stanford veterinary staff (Edward Florendo and Yvonne Zuber) for coordination of animal facility space used for behavioral experiments.

Correspondence should be addressed to Meng-meng Fu at mengmengfu@ gmail.com.

https://doi.org/10.1523/ENEURO.0170-20.2020

Copyright $@ 2020$ Nguyen et al.

This is an open-access article distributed under the terms of the Creative Commons Attribution 4.0 International license, which permits unrestricted use, distribution and reproduction in any medium provided that the original work is properly attributed.
MYRF (myelin regulatory factor) was knocked out in oligodendrocyte precursor cells (OPCs) display long-term defects in freezing (Pan et al., 2020). In addition, loss of the developmental regulator Cdk5 (cyclin-dependent kinase 5 ) in mature oligodendrocytes reduces myelination and fear-based learning (Luo et al., 2018). However, as ERK, CDK5, and MYRF broadly regulate cell function, it is unclear whether structural myelin changes underlie these changes.

Our study mechanistically illuminates the relationship between myelination and fear-based behaviors using a mouse in which hypomyelination has been attributed to structural defects in microtubules. Oligodendrocytes contain two classes of microtubules, radial microtubules that are proximal to the cell body and extend toward axons, and lamellar microtubules that are distal and spiral around the myelin sheath (Weigel et al., 2020). Oligodendrocytes rely on the formation or nucleation of microtubules outside the cell body off of Golgi outpost organelles (Valenzuela et al., 2020). This nucleation function is performed by tubulin polymerization promoting protein (TPPP; Fu et al., 2019). Tppp mRNA is highly expressed by oligodendrocytes, but extremely low in other brain cells (Zhang et al., 2014; Schaum et al., 2018) and in other non-brain tissues (Schaum et al., 2018).

A previous study found that both cultured and in vivo oligodendrocytes from homozygous Tppp knock-out (KO) mice have shorter lamellar microtubules and consequently shorter and thinner myelin sheaths (Fu et al., 2019). Cultured Tppp KO oligodendrocytes have additional aberrant features, including more proximal branches, mixed microtubule polarity and accumulation of myelin basic protein $(\mathrm{Mbp})$ mRNA, an abundantly transported cargo (Carson et al., 1997; Herbert et al., 2017). Furthermore, immunostaining against MBP protein shows that Tppp KO brains have decreased myelination in spinal cord, caudate, cortex, and hippocampus, with upper cortical layers and CA1 and CA3 fiber tracts that are strikingly devoid of myelination. No gross differences were observed in neurofilament staining, indicating that axonal tracts and neuronal morphology is largely intact in Tppp KO mice (Fu et al., 2019).

Our current research explores fear-based behaviors in these myelin-deficient mice. In open-field assays and lightdark preference assays, Tppp KO mice display similar activity levels as wild-type mice, indicating lack of anxiety behavior. In contrast, Tppp KO mice are profoundly deficient in fear responses. We use two types of assays, traditional fear conditioning and looming fear, to evaluate memory-dependent fear responses and innate fear responses, respectively. Results from both fear conditioning and spatial memory tests support short-term memory deficits in Tppp KO mice. Altogether, our study further strengthens the connection between myelination and fear-based learning and adds an additional connection to innate fear.

\section{Materials and Methods}

\section{Animals}

All animal procedures were performed in accordance with the Stanford University animal care committee's 
regulations. Tppp KO mice were recovered from sperm from the Knockout Mouse Project (KOMP; stock number VG12652) and bred in the C57BL/6J strain. Animals were bred from heterozygous parents and age-matched littermates were selected for behavioral assays. Only threemonth-old male mice were used for behavioral experiments. Mice that underwent fear conditioning were subject either to this assay alone or after all other behavioral tests were completed. Mice were tested blinded and the genotypes of mice were only revealed to the experimenter after the end of the experiment. All data represent one observation per animal per assay as additional consecutive trials would reflect habituation rather than an independent biological replicate.

\section{Open field activity assay}

An individual mouse was placed in a new cage inside the SmartCage platform (AfaSci) for $1 \mathrm{~h}$. The animal's position and locomotion were determined by breakages in the infrared (IR) beams provided by the SmartCage platform. Measurements of activity counts (i.e., breaks in the IR beams in the $x-, y$-, and/or $z$-directions) were automatically quantified using the accompanying CageScore software (Khroyan et al., 2012).

\section{Light-dark box assay}

To assess anxiety-related behavior, a dark red box made of Plexiglas with a small entrance was placed inside the mouse cage and extra overhead lighting was provided outside this box, separating the cage into a light compartment and a dark compartment. The amount of time the animal spent in the light or dark compartment was quantified by CageScore (Khroyan et al., 2012) over a period of $1 \mathrm{~h}$.

\section{Trace fear conditioning}

Individual mice were placed inside the fear conditioning chamber (Coulbourn Instruments) which was cleaned with $10 \%$ ethanol as background odor. A ventilation fan provided $\sim 55 \mathrm{~dB}$ of background noise. Mice were preexposed to the chamber for $5 \mathrm{~min} 24 \mathrm{~h}$ before training. On training day (day 1, $24 \mathrm{~h}$ later), after a 4-min period of exploration to establish baseline behavior, a $85-\mathrm{dB} 2-\mathrm{kHz}$ tone was played for $20 \mathrm{~s}$ (cue period), followed by an 18-s trace period and a 2-s foot shock of $0.75 \mathrm{~mA}$. The process was repeated four times and separated by 1 -min intertrial intervals (ITIs).

On context recall day (day 2, $24 \mathrm{~h}$ later), mice were placed back in the training chamber for $4 \mathrm{~min}$. On altered context testing day (day 3, $24 \mathrm{~h}$ later), mice were placed in an altered conditioning chamber (side walls decorated with different patterns, smooth solid plastic floor, $1 \%$ vanilla background odor); the same protocol used on training day minus the foot shock was applied. The behavior of the mice was recorded and analyzed with FreezeView software (Coulbourn Instruments). On training day, the freezing percentages during each exploration, cue, trace, and ITI period were summarized as an indication of fear memory acquisition. On context recall day, the freezing percentage was compared with that of the last ITI of training day. On altered context testing day, the averaged freezing percentages of cue, trace, and ITI periods were reported.
Fear-conditioned mice were not subsequently used for any other assays.

\section{Y-maze}

To assess working spatial memory, an acrylic $Y$-shaped maze consisting of three symmetrical arms $(20 \times 8 \times$ $16 \mathrm{~cm})$ at $120^{\circ}$ angles was used as the testing arena. The end of each arm of the maze was decorated with a different visual cue. Individual mice were placed at the center of the maze and allowed to freely explore the three arms for $5 \mathrm{~min}$. Over the course of the testing time, wild-type mice are expected to display a propensity to explore a new arm of the maze instead of re-entering a previously visited arm. Arm entry was scored when the animal reached the end of the arm of the maze. The total number of entries and the number of unique triad combination of consecutive arm entries (ABC, ACB, BAC, BCA, CAB, $\mathrm{CBA}$ ) were manually scored. The percentage of alteration (spontaneous alteration) was calculated as the number of unique entries/(total number of entries - 2).

\section{Looming fear assay}

Looming behavior was measured as previously described (Salay et al., 2018). Briefly, a mouse was placed in a $50 \times 25 \times 40 \mathrm{~cm}$ glass chamber with a $12-\mathrm{cm}$ shelf at one end of the chamber to provide a shelter for mice to hide. At the top of the cage, a 24-inch LCD monitor facing onto the cage displayed stimuli. A video camera was placed outside the cage to record the mouse's movements. Mice were introduced to the cage and allowed to habituate for $\sim 10 \mathrm{~min}$. Then a looming stimulus (expanding black disk on a white background, 15 expansions over 24 s) was projected on the LCD monitor.

Video recordings were analyzed by bins of $1 \mathrm{~s}$. The percentage of time spent hiding, freezing, running, or ambulating was calculated. Hiding was classified as the mouse's entire body being underneath the shelf. Freezing was classified as episodes of $>3 \mathrm{~s}$ of no movement except for respiration. Running was classified as greater than two times the average ambulatory speed. All other motile behaviors were classified as ambulation.

The number of rearing events and head tilts were individually scored (i.e., they are not part of the percentage calculations above). Head tilts were defined as raising the head $>20^{\circ}$ (relative to the main body axis with vertex at the nape of the neck).

\section{Pupillary light reflex (PLR)}

Mice were dark-adapted for $1 \mathrm{~h}$ before measurement. Mice were manually restrained and placed in front of a video camera. After $5 \mathrm{~s}$, blue light was placed in front of the left pupil for $35 \mathrm{~s}$, after which the pupils were allowed to recover. Pupil size was analyzed using ImageJ and normalized to total eye size.

\section{Novel female interest assay}

As previously described (Jones et al., 2019), a cardboard box was used as the testing arena for female location memory test. On day 0, $24 \mathrm{~h}$ before female exposure, 
A

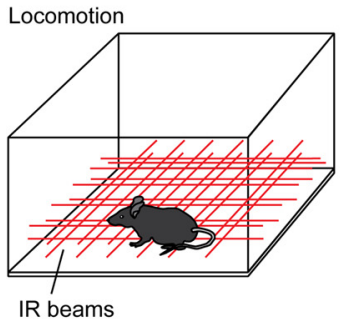

D

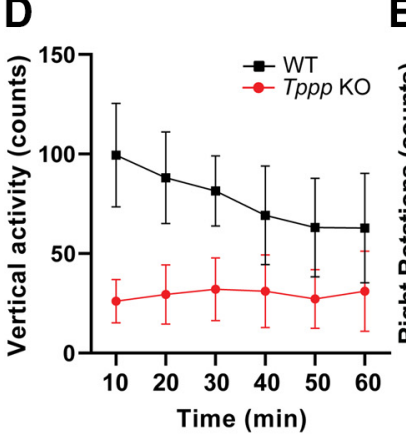

B

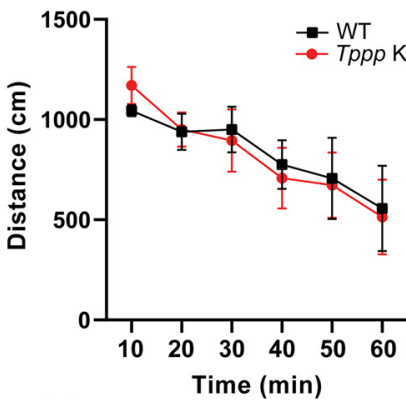

$E$

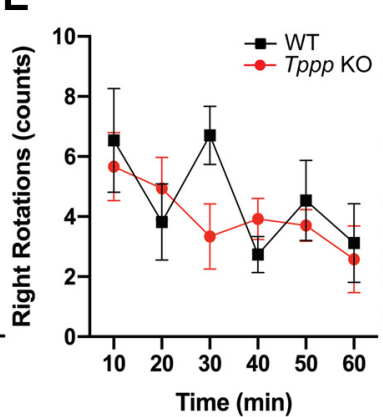

C

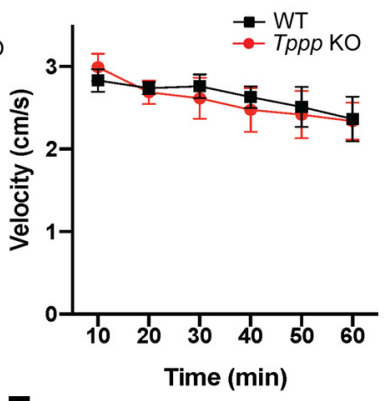

$\mathbf{F}$

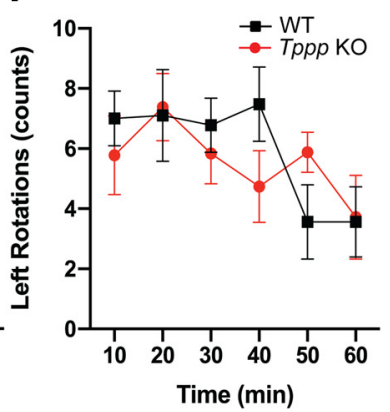

Figure 1. Tppp KO mice display normal open field activity. A, Open field assay experimental design. Wild-type and Tppp KO mice were allowed to move freely through an arena where IR beams measured their movement; $n=5$ mice per genotype for all data in this figure. Detailed statistical results can be found in Extended Data Figure 1-1; power analyses can be found in Extended Data Figure 1-2. B, Wildtype and Tppp KO mice did not show any differences in distance traveled during the 60-min locomotion test. $\boldsymbol{C}$, Wild-type and Tppp KO mice did not show any differences in velocity during the 60-min locomotion test. $\boldsymbol{D}$, Wild-type and Tppp KO mice did not show any significant differences in number of vertical activity events when evaluated by two-way ANOVA ( $p=0.170$, time $\times$ genotype interaction). Evaluating wild-type versus Tppp KO means at each time point using independent $t$ tests yielded $p=0.016$ for the first time point (wild type: 99.4 counts, Tppp KO: 26.0 counts) but $p>0.05$ for all other timepoints, suggesting that wild-type mice show significantly more vertical activity during the first $10 \mathrm{~min}$ of the 60 -min locomotion test. Graph represent significance based on two-way ANOVA with post hoc Sidak's multiple comparisons test. $\boldsymbol{E}$, Wild-type and Tppp KO mice did not show any significant differences in number of right rotations during the 60 -min locomotion test. At the 30-min time point, $p=0.209$ ( $p=0.0504$ by independent $t$ test of means at that time point). $\boldsymbol{F}$, Wild-type and Tppp KO mice did not show any differences in number of left rotations during the 60-min locomotion test. At the 40-min time point, $p=0.4741$ ( $p=0.149$ by independent $t$ test of means at that time point) and at the 50 -min time point, $p=0.6586$ ( $p=0.148$ by independent $t$ test of means at that time point). At all other time points, $p>0.45$. Error bars represent SEMs.

individual male mice were allowed to freely explore the testing arena for $10 \mathrm{~min}$. On day 1 (experience day), a novel female mouse (unfamiliar to the male mice) was randomly placed inside one of the four slotted metallic enclosures placed at the corners of the testing arena. Male mice were placed at the center of the arena facing the visual cues on the wall and subjected to $10 \mathrm{~min}$ of experience trial. The amount of time the male mice spent interacting with the female inside the enclosure was scored as female interest.

\section{Statistical analysis}

All data are presented as mean \pm SEM (standard error of the mean) and were analyzed with Prism 8 (GraphPad Software). Data were evaluated for normality using Kolmogorov-Smirnov test. For data with two conditions, parametric data were analyzed using unpaired $t$ test; nonparametric data were analyzed using Mann-Whitney (Wilcoxon rank-sum test). All other data were analyzed using two-way ANOVA (analysis of variance) with post hoc Sidak's multiple comparison test to compare wild-type to Tppp KO mice values; $p<0.05$ was considered significant, and the following notations were used to indicate significance: $* p<0.05, * * p<0.01, * * * p<0.001, * * * * p<0.0001$.

\section{Results}

\section{Open field and light-dark box tests}

We first examined the baseline activity level of Tppp KO mice using a 60-min open field test in which intersecting IR beams were used to measure lateral and vertical translocation (Gould et al., 2009; Fig. 1A). There was no significant difference in total horizontal distance or velocity traversed by Tppp KO mice compared with their wild-type counterparts (Fig. 1B,C; Extended Data Figs. 1-1, 1-2), indicating similar levels of ambulation and activity. Tppp KO mice did not show significant differences in vertical activity (Fig. 1D). No differences in the number of right or left rotations were observed between Tppp KO mice and wild-type mice (Fig. 1E,F; Extended Data Figs. 1-1, 1-2), indicating no evidence of aberrant twirling or spinning behaviors.

We also examined anxiety-like behavior in Tppp KO mice using a light-dark box test (Fig. 2A), in which anxious mice are expected to spend more time in darker areas than in lit areas (Crawley, 1985). There was no significant difference in the percentage of time spent in the dark zone when analyzed by 10 -min bins or by total time 
A

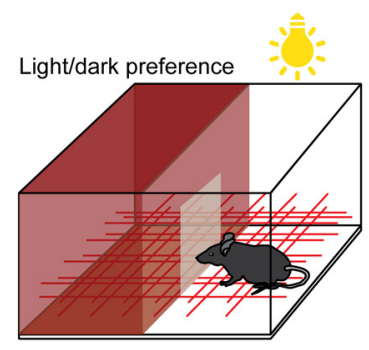

B

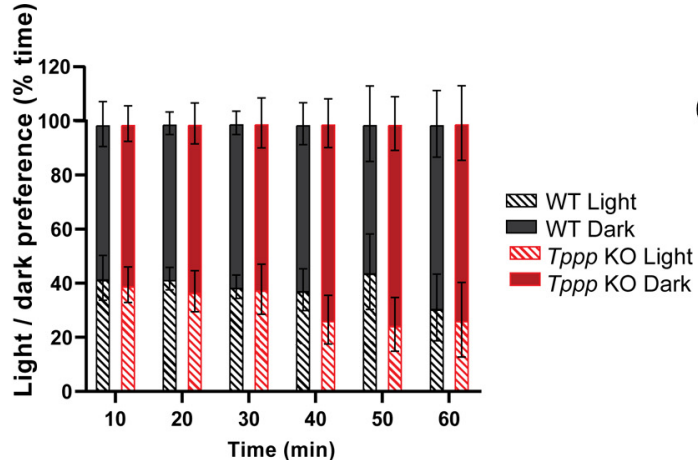

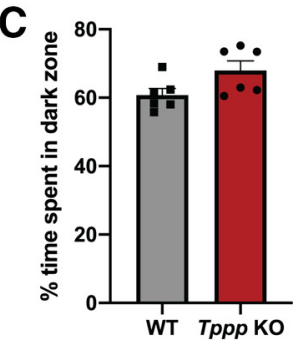

Figure 2. Tppp KO mice display normal light-dark preferences. $\boldsymbol{A}$, Light-dark box assay experimental design. Wild-type and Tppp KO mice were allowed to move freely between light and dark sides of a cage to examine anxiety; $n=5$ mice per genotype for all data in this figure. Detailed statistical results can be found in Extended Data Figure 2-1; power analyses can be found in Extended Data Figures 1-2, 2-2. B, Wild-type and Tppp KO mice did not show any difference in percentage of time spent in light or dark zones when analyzed in 10-min bins. $\boldsymbol{C}$, Wild-type and Tppp KO mice did not show any difference in total percentage of time spent in the dark zone. Error bars represent SEMs.

between Tрpp $\mathrm{KO}$ and wild-type mice (Fig. 2B,C; Extended Data Figs. 2-1, 2-2), suggesting normal levels of unconditioned or inherent anxiety in Tppp KO mice.

\section{Fear conditioning assay}

Having established normal anxiety and baseline activity levels, we examined differences in fear conditioning learning between Tppp $\mathrm{KO}$ and wild-type mice using a context-dependent and cue-dependent trace conditioning assay (Fig. $3 A$ ). On day 0 of this assay, mice were acclimated to a new environment. On day 1, mice were returned to this same environment, and their baseline activity was assessed. Our data indicate that Tppp KO and wild-type animals display similar levels of low or no baseline freezing (Fig. 3B), which is consistent with open field assays in which Tppp KO mice have similar levels of locomotion as wild-type mice (Fig. 1B). Animals are then exposed to an auditory cue followed by a noxious stimulus (foot shock). Although at $85 \mathrm{~dB}$ this tone is not loud enough to stimulate an acoustic startle response (Pantoni et al., 2020), both wild-type and Tppp KO mice increase the percentage of time spent freezing. This indicates that Tppp KO mice can perceive the tone (i.e., are not deaf) and furthermore that they freeze acutely in response to the tone. As four successive cycles of cue and shock proceed, both wild-type and Tppp KO mice respond by increasing the percentage of time spent freezing (Fig. 3B). Interestingly, looking individually at periods during the fourth and last cycle, Tppp KO mice surprisingly display slightly lower percentages of time spent freezing during the period following the final auditory cue (ITI4, by $t$ test), although these differences are not significant by two-way ANOVA (Fig. 3B; Extended Data Fig. 3-1).

On the following days, mice are assessed for contextbased recall and cue-based recall. On day 2 , mice are returned to the same environment, but with no auditory cue or foot shock (Fig. $3 A$ ) and context recall is measured by their fear response in terms of time spent freezing. Wildtype animals displayed similar levels of freezing (Fig. 3C) as day 1 during the last two cycles of training (Fig. $3 B$ ). Remarkably, on day 2, Tppp KO mice spent about half as much time frozen (25.8\%) compared with wild-type mice (53.2\%); this difference (27.4\% less) had a $95 \%$ margin of error of $9.1 \%$ (Fig. 3C). Interestingly, Tppp KO mice spent a similar percentage of time frozen as they did during the first cycle of shocks on day 1 (Fig. 3B). This indicates a significant degradation of the contextual memory in Tppp $\mathrm{KO}$ mice when they were returned to the conditioning chamber.

On day 3 , to evaluate cue-based recall, mice were introduced to a new environment but the auditory cue is played again for four cycles with the same timing as day 1 (Fig. 3A). These results were analyzed for the individual periods of each cycle (Fig. $3 D$ ) and as binned results for each period (Fig. 3E). Baseline freezing levels were lower in Tppp KO mice before the auditory cue (Fig. 3E), consistent with day 2 context recall results (Fig. $3 C$ ). During the cue recall, wild-type mice froze $\sim 40-50 \%$ of the time during the cue sound, during the trace period immediately following the cue, and during the ITI before the next cycle. In contrast, Трpp KO mice spend significantly less time freezing, only $\sim 10-15 \%$ (Fig. 3E). These differences are most profound during the first two cycles (Fig. 3D). Thus, the lack of a significant freezing response in Tppp $\mathrm{KO}$ mice after the auditory tone suggests a severe impairment in their cued memory recall.

Strikingly, Тppp KO mice froze significantly less than wild-type mice during all stages of this assay, initial fear conditioning on day 1 (Fig. 3B), context-based recall on day 2 (Fig. $3 C$ ), and cue-based recall on day 3 (Fig. $3 D, E$ ). Together, these results are consistent with a robust defect in fear conditioning.

We next asked whether the attenuated contextual fear memory retention in Tppp $\mathrm{KO}$ mice could be a result of spatial memory deficit using the $\mathrm{Y}$-maze test (Fig. 4A). We observed that wild-type mice and Tppp $\mathrm{KO}$ mice had no difference in total number of entries (Fig. 4B; Extended Data Fig. 4-1), perhaps consistent with similar distances traversed in the open field assay (Fig. 1B). However, Tppp $\mathrm{KO}$ mice had significantly lower percentage of unique 
A

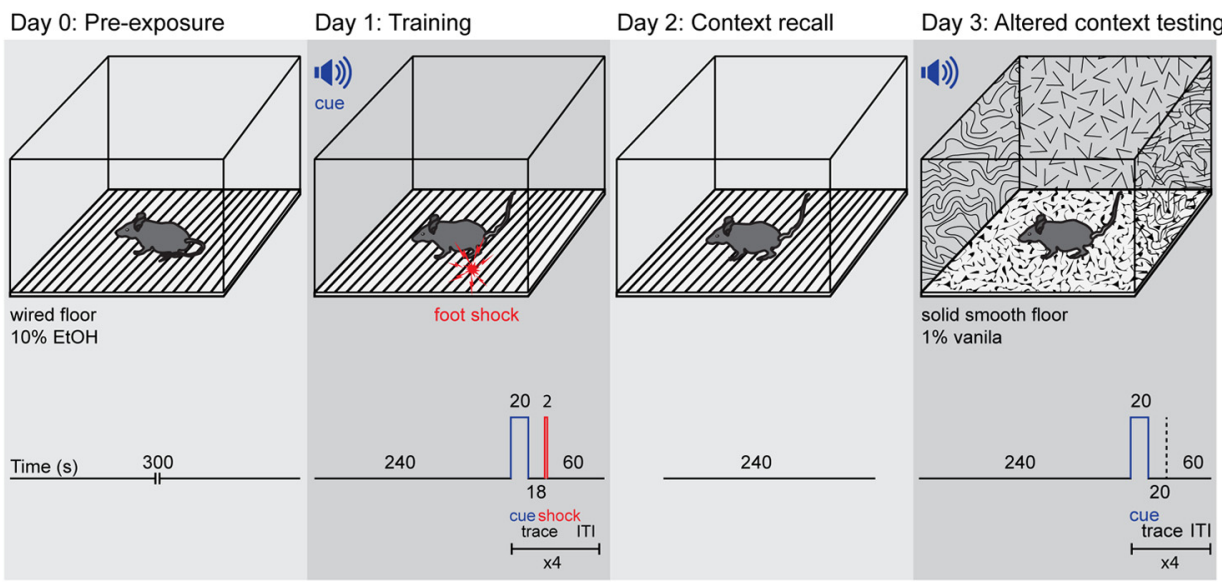

B

C
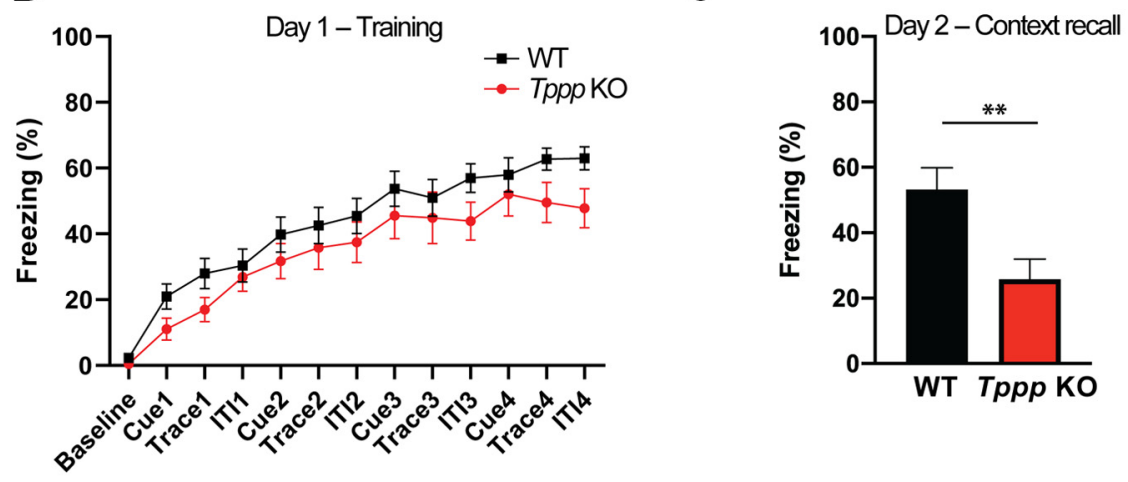

D

Day 3 - Altered context testing

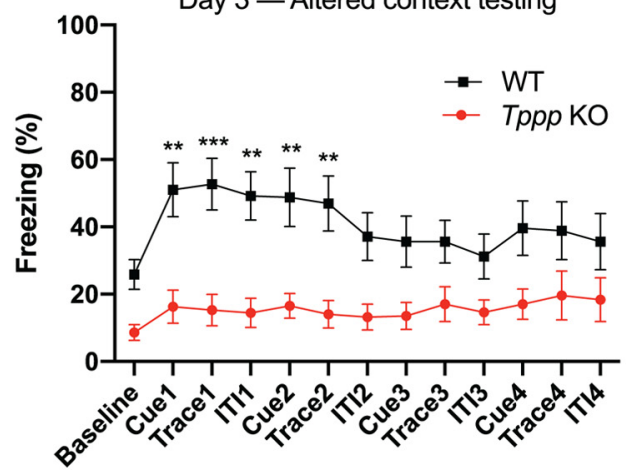

E

Day 3 - Altered context testing

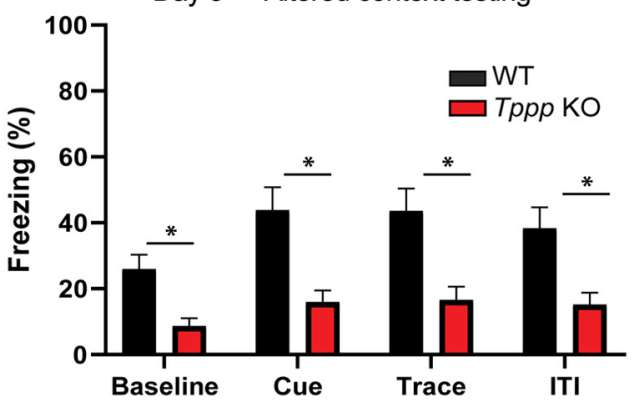

Figure 3. Tppp KO mice display defective fear conditioning. $\boldsymbol{A}$, Fear conditioning assay experimental design. On day 0 , mice were acclimated to a wired-floor cage with a background scent of $10 \%$ ethanol. On day 1, the mice were again acclimated to the cage and then a cue sound was played for $20 \mathrm{~s}$ followed by a foot shock and a 60-s ITI. The cue, trace, shock, and ITI were repeated for a total of four times. On day 2, mice were placed in the same wired floor cage with $10 \%$ ethanol scent. On day 3 , mice were acclimated to a cage with solid smooth flooring and $1 \%$ vanilla background scent and then given the cue sound, without the shock; $n=11$ mice per genotype for all data in this figure. Detailed statistical results can be found in Extended Data Figure 3-1; power analyses can be found in Extended Data Figures 1-2, 2-2. B, On day 1 (training), Tppp KO mice had similar freezing responses as wildtype mice, with no significant differences in percentage time spent freezing when evaluated by two-way ANOVA with post hoc Sidak's multiple comparisons test. Graphs represent significance based on two-way ANOVA with post hoc Sidak's multiple comparisons test. Evaluating wild-type versus Tppp KO means as independent $t$ tests (i.e., at different time points/periods) gives $p$ values of 0.371 (wild type: $2.306 \%$, Tppp KO: $0.472 \%$ ) at baseline and of 0.042 (wild type: $62.94 \%$, Tppp KO: $47.79 \%$ ) during ITI4 $(p=0.042)$. All other $p$ values generated by independent $t$ tests were $>0.05$. $C$, On day 2 (context recall), Tppp KO mice displayed less freezing overall compared with wild-type mice. $p=0.007$ by Welch's $t$ test. $\boldsymbol{D}$, On day 3 (context recall), Tppp KO mice displayed significantly less freezing than wild-type mice at all timepoints during the first two rounds of training. Graphs represent significance based on two-way ANOVA with post hoc Sidak's multiple comparisons test. Evaluating wild-type versus Tppp KO means as independent $t$ tests (i.e., at different time points/periods) reveals significant $p$ values for all other timepoints except trace 3: at baseline, $p=0.0014$ (wild type: $24.74 \%$, Tppp KO: $8.650 \%$ ); for cue 3, $p=0.0213$ (wild type: 35.65\%, Tppp KO: 13.55\%); for trace 3, 
continued

$p=0.0634$ (wild type: 35.64\%, Tppp KO: 17.06\%); for ITI 3, $p=0.0473$ (wild type: $31.21 \%$, Tppp KO: 14.60\%); for cue 4, $p=0.0269$ (wild type: 39.66\%, Tppp KO: 17.03\%); for trace 4, $p=0.0294$ (wild type: 38.85\%, Tppp KO: 19.64\%); for ITI 4, $p=0.0320$ (wild type: $35.63 \%$, Tppp KO: 18.36\%). E, On day 3, Tppp KO mice displayed significantly less freezing during baseline, cue, trace, and ITI periods than wildtype mice; $p$ values calculated by two-way ANOVA. Error bars represent SEMs. $* p<0.05, * * p<0.01, * * * p<0.001$.

entry combinations (i.e., spontaneous alternating behavior) than their wild-type counterparts (Fig. $4 C$ ), suggesting deficits in working spatial memory.

\section{Looming fear assay}

We next sought to understand whether Tppp KO mice also have decreased innate fear response to natural threats using the looming fear assay. This assay tests innate visual fear response using a looming screen that projects a dark, rapidly expanding round object on a white background that simulates a swooping predator (Fig. 5A; Salay et al., 2018). We binned mouse behavior during the looming stimulus into freezing, hiding, running, and ambulating (Fig. 5B-E; Extended Data Fig. 5-1; Movies 1,2). While wild-type mice spent the majority of time hiding (76.3\%), Tppp KO mice spent significantly less time hiding (15.8\%); this difference of $60.5 \%$ had a $95 \%$ margin of error of $9.1 \%$ (Fig. $5 C$ ). In contrast, Tppp $\mathrm{KO}$ mice spent the majority of time ambulating (67.8\%), which was significantly higher than in wild-type mice (14.3\%); this difference of $53.4 \%$ had a $95 \%$ margin of error of $15.6 \%$ (Fig. $5 D$ ). No statistically significant difference was observed for the percentage of time spent freezing, although we calculated a low power for this comparison, possibly indicating a false negative result (Fig. 5E; Extended Data Fig. 2-2).

In addition, we observed incidents of rearing and head tilting in Tppp KO mice and quantified these in terms of number of events. Surprisingly, Tppp KO mice also had significantly more rearing events when compared with wild-type mice, in which only one rearing event was observed across all animals (Fig. 5F). In addition, Tppp KO mice also displayed more head tilting events (15.2) compared with wild-type mice (4.4); this difference (10.8 more) had a 95\% margin of error of 2.2 (Fig. 5G). Both rearing and head tilting behavior appear to be attempts to look at the looming stimulus. Together, results from this assay suggest a robust deficit in innate fear response in Tppp KO mice.

Visual defects can have a significant impact on the outcomes of behavioral tests. For example, an alternate interpretation to the looming assay result could be that Tppp $\mathrm{KO}$ mice are not able to visually perceive the looming stimulus. To rule out the possibility of a vision deficit, we used the PLR test to measure the pupil dilation response on the contralateral eye (Fig. 6A). Our results show that Tppp KO mice display a PLR response with no significant differences compared with their wild-type counterparts (Fig. 6B; Extended Data Fig. 6-1). These results show that Tppp KO mice dilate pupils following exposure to high amounts of light and suggest that Tppp KO mice are not grossly visually impaired.

To further assess whether Tppp KO mice have sensory or social deficits, we introduced them to the novel female interest assay (Fig. 6C). In this paradigm, mice rely on visual and olfactory cues to detect the presence of the novel female. Tppp KO mice displayed no difference from wildtype animals in interaction time (Fig. $6 D$; Extended Data Fig. 6-1). Thus, this indicates that Tppp KO mice are neither visually or sensorially impaired in their detection of the novel female nor socially impaired in their interest in the novel female.

\section{Discussion}

By performing a number of behavioral assays, we show that the hypomyelinated Tppp KO mouse displays a specific subset of behavioral deficits. In open-field and lightdark preference assays, Tppp KO mice display similar activity levels and time spent in the light and dark compartments compared with wild-type mice, indicating that they do not display anxiety behavior. However, in both
A

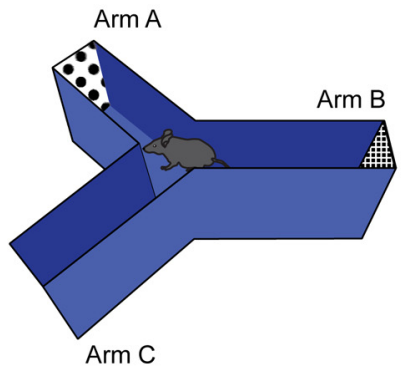

B

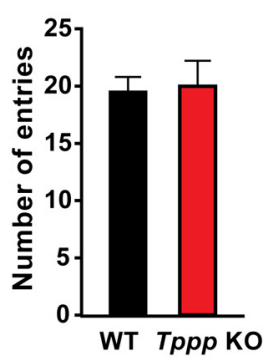

C

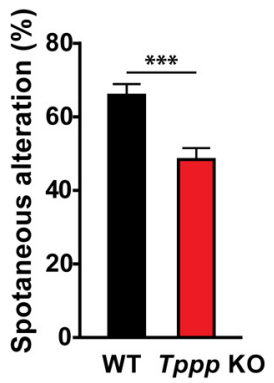

Figure 4. Tppp KO mice have spatial memory defects. $\boldsymbol{A}$, Y-maze assay experimental design. Wild-type and Tppp KO mice were placed in the center of a Y-shaped apparatus and allowed to travel to one of its arms, each with a different visual cue at the end; $n=8$ mice per genotype for all data in this figure. Detailed statistical results can be found in Extended Data Figure 4-1; power analyses can be found in Extended Data Figure 2-2. B, Tppp KO and wild-type mice did not show a significant difference in number of total entries. C, Tppp KO mice had lower percentage of spontaneous entry alteration than wild-type counterparts; $p=0.0014$ by Welch's $t$ test. Error bars represent SEMs. $* * * p<0.001$. 


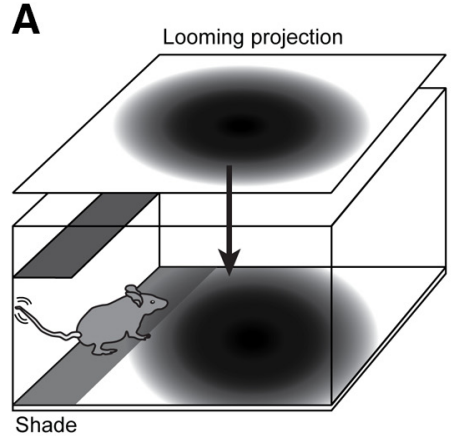

C

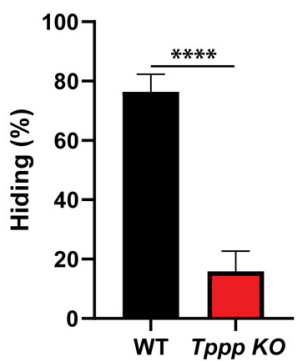

$F$

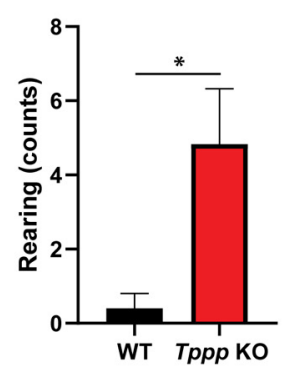

B

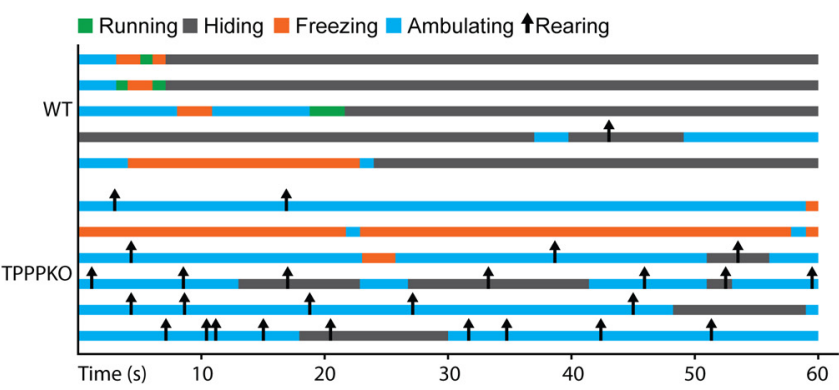

D

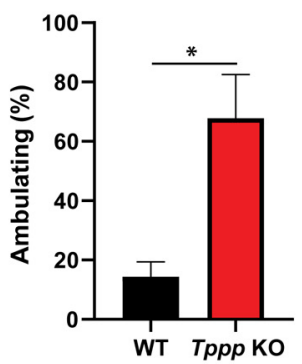

E

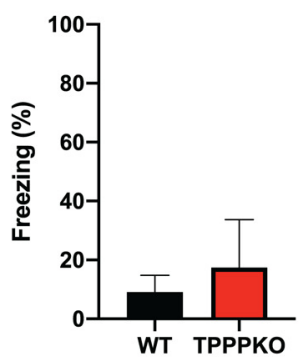

G

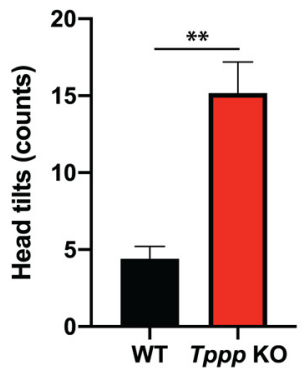

Figure 5. Tppp KO mice lack looming fear response. A, Looming fear assay experimental design. Mice were placed in an arena with a shelf on one side to hide under. The ceiling of the arena contained an LED screen that projected a looming stimulus, an expanding black circle on a white background, onto the cage. Detailed statistical results can be found in Extended Data Figure 5-1; power analyses can be found in Extended Data Figure 2-2. B, Ethogram of responses to looming stimulus, classified as either running, hiding, freezing, or ambulating. Arrows represent rearing events. Running events were only observed in wild-type mice; $n=5$ wild-type mice, $n=6$ Tppp KO mice for all data in this figure. $\boldsymbol{C}-\boldsymbol{E}$, Percentage of time spent hiding, ambulating or freezing in response to looming stimulus. Compared with wild-type mice, Tppp KO mice spent significantly less time hiding $(p=0.0001)$, significantly more time ambulating $(p=0.011)$, and no significant difference in time freezing $(p=0.26)$. $\boldsymbol{F}$, Tppp KO mice displayed significantly more rearing events than wild-type mice $(p=0.030)$. $\mathbf{G}$, Tppp KO mice displayed significantly more head tilts than wild-type mice. Error bars represent SEMs. $* p<0.05, * * p<0.01, * * * * p<0.0001$.

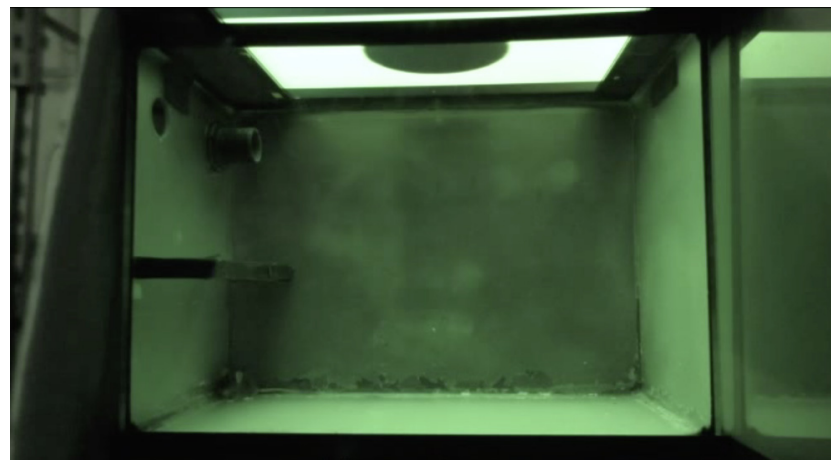

Movie 1. Looming fear in a wild-type mouse. Video recording of looming fear assay performed on a wild-type mouse. [View online]

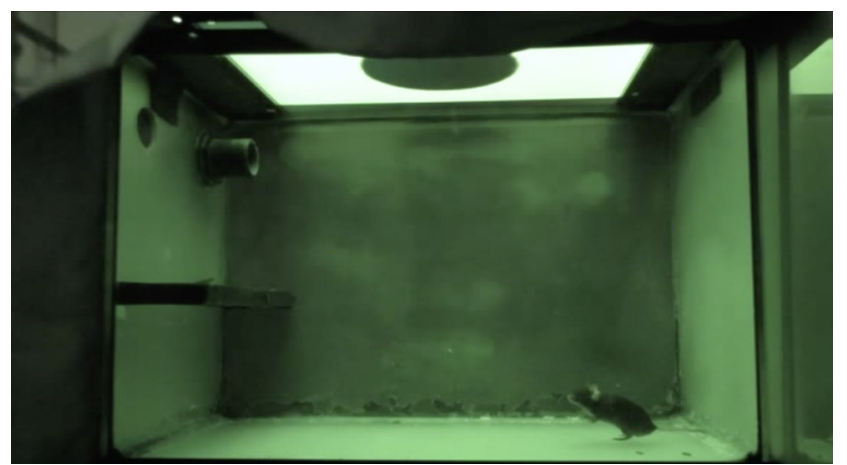

Movie 2. Looming fear in a Tppp KO mouse. Video recording of looming fear assay performed on a Tppp KO mouse. [View online] 
A

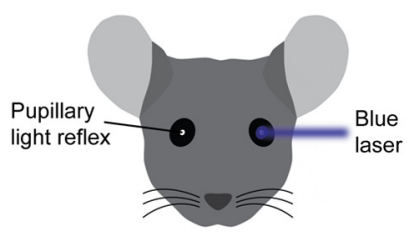

B

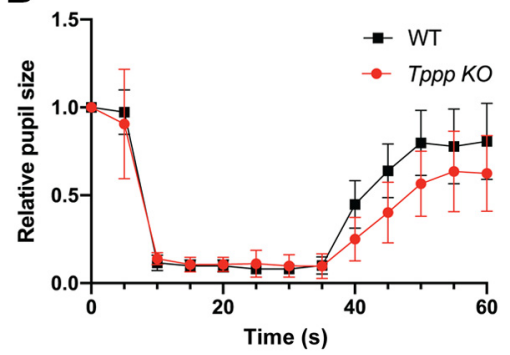

C

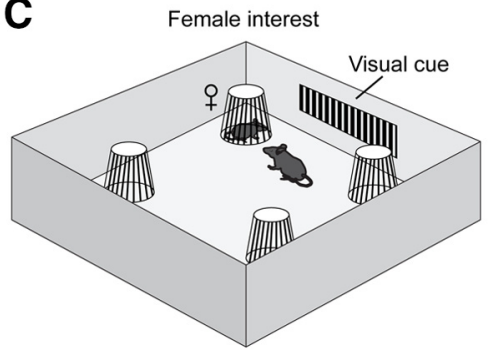

D

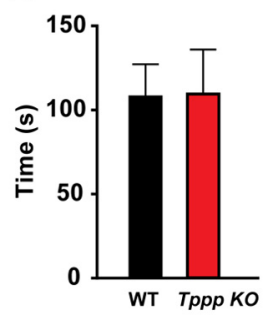

Figure 6. Tppp KO mice have normal pupillary dilation. A, Pupillary dilation assay experimental design. A blue laser was beamed into the left pupil and the light reflex of the right pupil was recorded. Detailed statistical results can be found in Extended Data Figure 6-1; power analyses can be found in Extended Data Figures 1-2, 2-2. B, Both wild-type and Tppp KO mice display pupil dilation reflexes. Graphs represent significance based on two-way ANOVA with post hoc Sidak's multiple comparisons test, which shows no significant difference in relative pupil size between Tppp KO and wild-type mice. Evaluating wild-type versus Tppp KO means as independent $t$ tests (i.e., at different time points/periods) gives significant $p$ values at 40 and $45 \mathrm{~s}$ ( $p=0.025$ at $40 \mathrm{~s}$ and $p=0.030$ at $45 \mathrm{~s})$, but does not show significant differences at other timepoints $(p=0.055$ at $50 \mathrm{~s}$ and $p>0.15$ at all other timepoints); $n=6$ mice per genotype. $\boldsymbol{C}$, Novel female interest assay experimental design. A novel female mouse (unfamiliar to the male mice) was randomly placed inside one of the four slotted metallic enclosures placed at the corners of the testing arena. Male mice were placed at the center of the arena facing the visual cues on the wall and subjected to 10 min of experience trial. $\boldsymbol{D}$, Female interest was scored as the amount of time (out of $10 \mathrm{~min}$ total) that the male mouse spent interacting with the female inside the enclosure; $n=8$ mice per genotype. Error bars represent SEMs.

traditional fear-conditioning assays and looming fear assays, Tppp KO mice show marked defects in both fear memory and innate fear, respectively. In addition, spatial memory tests support a short-term memory deficit in Tppp KO mice. These results are consistent with deficits in fear-based responses, but not in anxiety behavior.

Intriguingly, both learning-based fear and innate fear are altered in Tppp KO mice. Defects in learning-based fear are consistent with decreased MBP staining in the cortex and hippocampus as well as shorter sheath lengths in the cortex (Fu et al., 2019). However, the looming fear assay is non-memory dependent, and therefore, these deficits may indicate a myelination defect in the underlying innate fear circuitry. Freezing and hiding in response to visual looming threat involve activation of projections from the ventral midline thalamus (vMT) to the basolateral amygdala (BLA; Salay et al., 2018). Two major pathways connect the amygdala to other brain regions: stria terminalis, an efferent pathway to the basal forebrain and hypothalamus, and the amygdalofugal pathway, containing both afferent and efferent connections with the thalamus as well as with basal forebrain and hypothalamus. In contrast to the stria terminalis, the amygdalofugal pathway is a myelinated white matter tract (Mori et al., 2017). Anatomical patterns of myelination in the amygdalofugal pathway, such as average sheath length, frequency and lengths of unmyelinated axon segments, have yet to be elucidated. This type of data could provide mechanistic insights on why hypomyelinated Tppp KO animals display innate fear defects.

Indeed, structural deficits in myelination may affect conduction velocity and do so through several mechanisms, including regulation of myelin sheath length, thickness, or nodal length (Arancibia-Cárcamo et al., 2017). Tppp KO oligodendrocytes have shorter sheath lengths by about half when compared with wild-type oligodendrocytes both in vitro in 3D cultures as well as in vivo in the cortex (Fu et al., 2019). Furthermore, oligodendrocytes cultured from different regions, such as cortex and spinal cord, have intrinsic differences in sheath length (Bechler et al., 2015). Thus, brain regions with characteristically long myelin sheaths may be more adversely affected in Tрpp KO mice. In the amygdalofugal pathway, if myelin sheaths are characteristically long, then shorter sheath lengths in Tppp KO mice could contribute to looming fear defects.

Our observations of short-term fear memory deficits in Tppp KO mice are consistent with recent reports connecting myelination to long-term fear memory deficits. In wildtype mice, fear learning induces proliferation of OPCs in the medial PFC (mPFC), which then maturate over the time course of approximately four weeks. Mice in which the transcription factor MYRF was inducibly and conditionally knocked out in NG2-positive OPCs display striking defects in freezing in fear conditioning assays on the timescale of $30 \mathrm{~d}$ postconditioning, but not at $1 \mathrm{~d}$ 
postconditioning (Pan et al., 2020). This result was independently replicated with similar dramatic defects in freezing when tested $28 \mathrm{~d}$ following initial training. Mechanistically, local field potential recordings indicate increases in synchronization in the hippocampal-cortical circuit following fear learning, which is deficient in mutant mice (Steadman et al., 2020). These studies indicate that long-term memory consolidation involves oligodendrogenesis and new myelin sheaths. In contrast, Tppp KO mice display short-term fear memory deficits on the faster timescale of 1-2 d, likely consistent with preexisting structural defects in myelination.

Interestingly, other mouse models with structural myelin defects also display behavioral issues. CNP forms the "struts" of cytoplasmic channels that spiral around the myelin sheath (Snaidero et al., 2017) and contain lamellar microtubules (Snaidero et al., 2014). Compared with wildtype mice, heterozygous Cnp-null mice spend $>3$ times the amount of time holding on to a bar, in an assay that models catatonia, a symptom in schizophrenia. However, unlike Tppp KO mice (Fu et al., 2019), heterozygous Cnpnull mice do not display defects in Rotarod or fear conditioning (Hagemeyer et al., 2012). In addition, behavioral assays performed on mice lacking PLP (proteolipid protein), a transmembrane myelin protein, demonstrated that, in terms of motor coordination, Plp-null mice display no Rotarod deficits, but have gait and swimming defects. Behaviorally, Plp-null mice spend more time in the open arm of the zero maze and less time burying marbles, but, like Tppp KO mice, they displayed no open field assay differences. This specific subset of phenotypes can be attributed to regionally specific enhanced proliferation, as observed in the olfactory bulb and rostral corpus callosum (Gould et al., 2018). Thus, different mouse models of hypomyelination can display distinct subsets of defects; this indicates that future studies should not only perform multiple behavioral assays but should also consider regionally distinct effects in the brain.

Since individual tasks rely on many different brain regions, it is difficult to pinpoint the most affected regions. In PIp-null mice, task-specific differences may be attributed to regionally specific increases in oligodendrocyte density in the olfactory bulb and rostral corpus callosum (Gould et al., 2018). In Tppp KO mice, hypomyelination has been observed in many regions, including cortex, hippocampus, caudate, and spinal cord (Fu et al., 2019). Thus, TPPP deficiency may broadly affect oligodendrocyte function and contribute to multiple behavioral problems found in Tppp KO mice.

The emerging principle of myelin plasticity or adaptive myelination encompasses structural myelin changes following neuronal activity. This has been tested in many paradigms, both after learning and with social environment change. In long-term fear-based learning, oligodendrogenesis and increased myelination play a role in spatial learning and fear-based learning paradigms for periods of up to $30 \mathrm{~d}$ (Pan et al., 2020; Steadman et al., 2020; Wang et al., 2020). Mechanistically, learning can strengthen hippocampal-cortical circuits, likely via synchronization of impulse conduction (Freeman et al., 2016; Steadman et al., 2020). For motor learning, both mice subject to optogenetic stimuli and complex wheel activity display changes in myelin structure (Gibson et al., 2014; McKenzie et al., 2014; Xiao et al., 2016). In addition, mice housed in social isolation demonstrate changes in white matter (Liu et al., 2012) and mice subject to chronic social stress have decreased expression of oligodendrocyte genes and myelination (Lehmann et al., 2017; Cathomas et al., 2019). Thus, myelin plasticity may be crucial for behavioral responses to environmental changes and future studies should investigate the molecular involvement of TPPP in microtubule-based myelin structural in response to learning.

\section{References}

Arancibia-Cárcamo IL, Ford MC, Cossell L, Ishida K, Tohyama K, Attwell D (2017) Node of Ranvier length as a potential regulator of myelinated axon conduction speed. Elife 6:e23329.

Aston C, Jiang L, Sokolov BP (2004) Microarray analysis of postmortem temporal cortex from patients with schizophrenia. J Neurosci Res 77:858-866.

Bechler ME, Byrne L, Ffrench-Constant C (2015) CNS myelin sheath lengths are an intrinsic property of oligodendrocytes. Curr Biol 25:2411-2416.

Carson JH, Worboys K, Ainger K, Barbarese E (1997) Translocation of myelin basic protein mRNA in oligodendrocytes requires microtubules and kinesin. Cell Motil 38:318-328.

Cathomas F, Azzinnari D, Bergamini G, Sigrist H, Buerge M, Hoop V, Wicki B, Goetze L, Soares S, Kukelova D, Seifritz E, Goebbels S, Nave KA, Ghandour MS, Seoighe C, Hildebrandt T, Leparc G, Klein H, Stupka E, Hengerer B, et al. (2019) Oligodendrocyte gene expression is reduced by and influences effects of chronic social stress in mice. Genes Brain Behav 18:e12475.

Crawley JN (1985) Exploratory behavior models of anxiety in mice. Neurosci Biobehav Rev 9:37-44

Fields RD (2008) White matter in learning, cognition and psychiatric disorders. Trends Neurosci 31:361-370.

Foong J, Maier M, Barker GJ, Brocklehurst S, Miller DH, Ron MA (2000) In vivo investigation of white matter pathology in schizophrenia with magnetisation transfer imaging. J Neurol Neurosurg Psychiatry 68:70-74.

Freeman SA, Desmazières A, Fricker D, Lubetzki C, Sol-Foulon N (2016) Mechanisms of sodium channel clustering and its influence on axonal impulse conduction. Cell Mol Life Sci 73:723-735.

Fu MM, McAlear TS, Nguyen $H$, Oses-Prieto JA, Valenzuela A, Shi RD, Perrino JJ, Huang TT, Burlingame AL, Bechstedt S, Barres BA (2019) The Golgi outpost protein TPPP nucleates microtubules and is critical for myelination. Cell 179:132-146 e14.

Georgieva L, Moskvina V, Peirce T, Norton N, Bray NJ, Jones L, Holmans P, MacGregor S, Zammit S, Wilkinson J, Williams $\mathrm{H}$, Nikolov I, Williams N, Ivanov D, Davis KL, Haroutunian V, Buxbaum JD, Craddock N, Kirov G, Owen MJ, et al. (2006) Convergent evidence that oligodendrocyte lineage transcription factor 2 (OLIG2) and interacting genes influence susceptibility to schizophrenia. Proc Natl Acad Sci USA 103:12469-12474.

Gibson EM, Purger D, Mount CW, Goldstein AK, Lin GL, Wood LS, Inema I, Miller SE, Bieri G, Zuchero JB, Barres BA, Woo PJ, Vogel H, Monje M (2014) Neuronal activity promotes oligodendrogenesis and adaptive myelination in the mammalian brain. Science 344:1252304

Gould EA, Busquet N, Shepherd D, Dietz RM, Herson PS, Simoes de Souza FM, Li A, George NM, Restrepo D, Macklin WB (2018) Mild myelin disruption elicits early alteration in behavior and proliferation in the subventricular zone. Elife 7:e34783.

Gould TD, Dao DT, Kovacsics CE (2009) The open field test. In: Mood and anxiety related phenotypes in mice, pp 1-20. Totowa: Humana Press. 
Gregg JR, Herring NR, Naydenov AV, Hanlin RP, Konradi C (2009) Downregulation of oligodendrocyte transcripts is associated with impaired prefrontal cortex function in rats. Schizophr Res 113:277-287.

Hagemeyer N, Goebbels S, Papiol S, Kästner A, Hofer S, Begemann M, Gerwig UC, Boretius S, Wieser GL, Ronnenberg A, Gurvich A, Heckers SH, Frahm J, Nave KA, Ehrenreich H (2012) A myelin gene causative of a catatonia-depression syndrome upon aging. EMBO Mol Med 4:528-539.

Hakak Y, Walker JR, Li C, Wong WH, Davis KL, Buxbaum JD, Haroutunian V, Fienberg AA (2001) Genome-wide expression analysis reveals dysregulation of myelination-related genes in chronic schizophrenia. Proc Natl Acad Sci USA 98:4746-4751.

Herbert AL, Fu MM, Drerup CM, Gray RS, Harty BL, Ackerman SD, O'Reilly-Pol T, Johnson SL, Nechiporuk AV, Barres BA, Monk KR (2017) Dynein/dynactin is necessary for anterograde transport of Mbp mRNA in oligodendrocytes and for myelination in vivo. Proc Natl Acad Sci USA 114:E9153-E9162.

Hof PR, Haroutunian V, Copland C, Davis KL, Buxbaum JD (2002) Molecular and cellular evidence for an oligodendrocyte abnormality in schizophrenia. Neurochem Res 27:1193-1200.

Houyoux N, Wattiez R, Ris L (2017) A proteomic analysis of contextual fear conditioned rats reveals dynamic modifications in neuron and oligodendrocyte protein expression in the dentate gyrus. Eur $\mathrm{J}$ Neurosci 46:2177-2189.

Jeffries MA, Urbanek K, Torres L, Wendell SG, Rubio ME, Fyffe-Maricich SL (2016) ERK1/2 Activation in Preexisting Oligodendrocytes of Adult Mice Drives New Myelin Synthesis and Enhanced CNS Function. J Neurosci 36:9186-9200.

Jones PH, Deng B, Winkler J, Zirnheld AL, Ehringer S, Shetty V, Cox M, Nguyen H, Shen WJ, Huang TT, Wang E (2019) Over-expression of miR-34c leads to early-life visceral fat accumulation and insulin resistance. Sci Rep 9:13844.

Khroyan TV, Zhang J, Yang L, Zou B, Xie J, Pascual C, Malik A, Xie J, Zaveri NT, Vazquez J, Polgar W, Toll L, Fang J, Xie X (2012) Rodent motor and neuropsychological behaviour measured in home cages using the integrated modular platform SmartCage ${ }^{\mathrm{TM}}$. Clin Exp Pharmacol Physiol 39:614-622.

Kubicki M, Park H, Westin CF, Nestor PG, Mulkern RV, Maier SE, Niznikiewicz M, Connor EE, Levitt JJ, Frumin M, Kikinis R, Jolesz FA, McCarley RW, Shenton ME (2005) DTI and MTR abnormalities in schizophrenia: analysis of white matter integrity. Neuroimage 26:1109-1118.

Lehmann ML, Weigel TK, Elkahloun AG, Herkenham M (2017) Chronic social defeat reduces myelination in the mouse medial prefrontal cortex. Sci Rep 7:46548.

Liu J, Dietz K, DeLoyht JM, Pedre X, Kelkar D, Kaur J, Vialou V, Lobo MK, Dietz DM, Nestler EJ, Dupree J, Casaccia P (2012) Impaired adult myelination in the prefrontal cortex of socially isolated mice. Nature neuroscience 15:1621-3.

Luo F, Zhang J, Burke K, Romito-DiGiacomo RR, Miller RH, Yang $Y$ (2018) Oligodendrocyte-specific loss of Cdk5 disrupts the architecture of nodes of Ranvier as well as learning and memory. Exp Neurol 306:92-104.

McKenzie IA, Ohayon D, Li H, de Faria JP, Emery B, Tohyama K, Richardson WD (2014) Motor skill learning requires active central myelination. Science 346:318-22.
Mori S, Kageyama Y, Hou Z, Aggarwal M, Patel J, Brown T, Miller MI, Wu D, Troncoso JC (2017) Elucidation of White Matter Tracts of the Human Amygdala by Detailed Comparison between HighResolution Postmortem Magnetic Resonance Imaging and Histology. Front Neuroanat 11:16.

Pan S, Mayoral SR, Choi HS, Chan JR, Kheirbek MA (2020) Preservation of a remote fear memory requires new myelin formation. Nat Neurosci 23:487-499.

Pantoni MM, Herrera GM, Van Alstyne KR, Anagnostaras SG (2020) Quantifying the acoustic startle response in mice using standard digital video. Front Behav Neurosci 14:83.

Salay LD, Ishiko N, Huberman AD (2018) A midline thalamic circuit determines reactions to visual threat. Nature 557:183-189.

Schaum N, Karkanias J, Neff NF, May AP, Quake SR, Wyss-Coray T, Darmanis S, Batson J, Botvinnik O, Chen MB, Chen S, Green F, Jones R, Maynard A, Penland L, Oliveira Pisco A, Sit RV, Stanley GM, Webber JT, Zanini F, et al. (2018) Single-cell transcriptomics of 20 mouse organs creates a Tabula Muris. Nature 562:367-372.

Snaidero N, Mobius W, Czopka T, Hekking LH, Mathisen C, Verkleij D, Goebbels S, Edgar J, Merkler D, Lyons DA, Nave KA, Simons M (2014) Myelin membrane wrapping of CNS axons by PI(3,4,5)P3dependent polarized growth at the inner tongue. Cell 156:277-90.

Snaidero N, Velte C, Myllykoski M, Raasakka A, Ignatev A, Werner HB, Erwig MS, Mobius W, Kursula P, Nave KA, Simons M (2017) Antagonistic Functions of MBP and CNP Establish Cytosolic Channels in CNS Myelin. Cell Rep 18:314-323.

Steadman PE, Xia F, Ahmed M, Mocle AJ, Penning ARA, Geraghty AC, Steenland HW, Monje M, Josselyn SA, Frankland PW (2020) Disruption of oligodendrogenesis impairs memory consolidation in adult mice. Neuron 105:150-164.e6.

Tkachev D, Mimmack ML, Ryan MM, Wayland M, Freeman T, Jones PB, Starkey M, Webster MJ, Yolken RH, Bahn S (2003) Oligodendrocyte dysfunction in schizophrenia and bipolar disorder. Lancet 362:798-805.

Uranova N, Orlovskaya D, Vikhreva O, Zimina I, Kolomeets N, Vostrikov V, Rachmanova V (2001) Electron microscopy of oligodendroglia in severe mental illness. Brain Res Bull 55:597-610.

Valenzuela A, Meservey L, Nguyen H, Fu M (2020) Golgi Outposts Nucleate Microtubules in Cells with Specialized Shapes. Trends in Cell Biology S0962892420301458.

Wang F, Ren SY, Chen JF, Liu K, Li RX, Li ZF, Hu B, Niu JQ, Xiao L, Chan JR, Mei $F$ (2020) Myelin degeneration and diminished myelin renewal contribute to age-related deficits in memory. Nat Neurosci 23:481-486.

Weigel M, Wang L, Fu M (2020) Microtubule organization and dynamics in oligodendrocytes, astrocytes, and microglia. Dev Neurobiol. Advance online publication. Retrieved April 23, 2020. doi: 10.1002/dneu.22753.

Xiao L, Ohayon D, McKenzie IA, Sinclair-Wilson A, Wright JL, Fudge AD, Emery B, Li H, Richardson WD (2016) Rapid production of new oligodendrocytes is required in the earliest stages of motorskill learning. Nat Neurosci 19:1210-1217.

Zhang Y, Chen K, Sloan SA, Bennett ML, Scholze AR, O'Keeffe S, Phatnani HP, Guarnieri P, Caneda C, Ruderisch N, Deng S, Liddelow SA, Zhang C, Daneman R, Maniatis T, Barres BA, Wu JQ (2014) An RNA-sequencing transcriptome and splicing database of glia, neurons, and vascular cells of the cerebral cortex. J Neurosci 34:11929-11947. 